\title{
COGENE plays up benefits, plays down risks
}

IN A surprise statement last Sunday, the foreign secretary of the Royal Society, Mr Michael Stoker, announced the lifting of the ban on reporting of what promises to be the most important meeting on the risks and benefits of recombinant DNA techniques since Asilomar, the conference held in California in 1975 during which scientists called for strict control of the use of recombinant DNA techniques.

COGENE, the Committee on Genetic Experimentation of the International Council of Scientific Unions (ICSU), had previously issued a directive that the whole proceedings at Wye College, Ashford, Kent, UK would be off the record. The directive was the result of what was felt to be unbalanced press coverage concerning recombinant DNA risks, particularly after Asilomar.

Dr Stoker, however, when opening the meeting, asked participants to "tear up that note". There had, he said, been a change of attitude on the organising committee. But the note had already had its effect-only three journalists were present and they were from specialist scientific magazines, including Nature.

We are therefore now able to report that COGENE is shifting its attention from risks to benefits. Supported by UNESCO and the World Health Organisation, it is planning to hold a series of two to three week teaching courses in recombinant DNA techniques in developing countries beginning in January 1980. COGENE chairman Dr Bill Whelan said: "We have the money and we just want to find a country that would appreciate our help." Recombinant DNA techniques could be of great benefit in Third World countries, Whelan believes, particularly in agriculture, where they could help in a rapid assessment of the molecular genetics of local crops.

Meanwhile, however, COGENE is slowing up its attempts to assess the risks of the technology. "We have run out of good ideas for risk assessment experiments", said Dr Anna-Marie Skalka, who chairs the COGENE subcommittee on risk assessment. However, Professor Mark Richmond, a member of the UK's Genetic Manipulation Advisory Group (GMAG) who will report on his own assessment experiments at the meeting, believes that the real problem is finding willing researchers to do the work. "I took it up to show willing", he said, but the work takes up time, lab space and people especially when there is a wide feeling in the scientific community that the risks are minimal. "If the UK Medical Research Council wants people to assess risks it may have to force them to do it", he said.

Robert Walgate

\section{GMAG states the position on 'self-closing'}

AT the end of last week, GMAG issued a statement designed to satisfy the "urgent need to clarify certain issues" and to remove "any uncertainty of ambiguity about present requirements". As expected some self-cloning experiments-those using Escherichia coli $\mathrm{K} 12$ and other well-characterised nonpathogenic laboratory strains of $E$. coli, Bacillus subtilis, Bacillus stearothermophilus and Saccharomyces cerevisiaeare exempted from statutory notification.

In general, however, self-cloning experiments are still considered to be within GMAG's remit. "The group is satisfied" says the statement "that many self-cloning experiments are without conceivable hazard", but it "interprets the definition of genetic manipulation to cover self-cloning experiments". It also acknowledges "that there has been uncertainty on this point and that some users have interpreted the definition to exclude such experiments". The definition of genetic manipulation used by GMAG is "the formation of new combinations of heritable material by the insertion of nucleic acid molecules produced, by whatever means outside the cell, into any virus, bacterial plasmid or other vector system so as to allow their incorporation into a host organism in which they do not naturally occur but in which they are capable of continued propagation".

In future, therefore, all self-cloning experiments with systems other than those specifically designated as exempt, should be notified to GMAG which "will advise on containment". Those working with exempt organisms are asked to provide voluntarily "a limited amount of information about their plans to help (GMAG) keep in touch with all developments in the field". This should include "species and strains, the place of work and the scientists responsible".

GMAG is also seeking to amend regulations to cover the use of "products of genetic manipulation which are capable of self-propagation". Voluntary notification of the use of these products is requested so that GMAG can advise on containment. However, exemptions from this will include the products of those self-cloning experiments which are exempt and the insertion of Xenopus genes coding for $28 \mathrm{~S}, 18 \mathrm{~S}$ and $5 \mathrm{~S}$ ribosomal RNAs into prokaryotic DNA. Users are invited to propose other products of recombinants which could be added to this list. Special consideration is also to be given to scaled up work involving the growth of self-propagating products in volumes of 10 litres or more. Arrangements for this type of work are still under discussion.

GMAG still feels unable, according to the statement, to accept some of the principles that the United States and other countries have adopted, following the NIH lead. Nevertheless it will study the NIH guidelines to see if there are some procedures which could be adopted in the UK in the hope that collaboration with other national authorities will bring about some uniformity of procedure.

GMAG's terms of reference are restated as advising all those concerned in any way with genetic manipulation, continually assessing new genetic manipulation techniques (and new containment methods) and advising on appropriate action. It is also required to "maintain appropriate contacts with relevant government departments, the Health and Safety Executive and the Dangerous Pathogens Advisory Group", and to keep records of containment facilities and the qualifications of biological safety officers.

\section{UK minister criticises COGENE meeting}

MR David Ennals, UK Secretary of State for Health and Social Security, expressed his concern last week at the original intention to ban reporting of this week's COGENE meeting. "Basically, I'm opposed to secret conferences", he told the genetic engineering subcommittee of the House of Commons Select Committee on Science and Technology. "Far too much in the field of science is done behind closed doors".

The subcommittee was holding its first public session after a recent visit to the US. Mr Ted Leadbitter cited a recent US survey which had found that of 18 countries using recombinant DNA techniques, the US and the UK had the most stringent regulations. Might this encourage valuable work to go abroad, he asked? Mr Ennals said he thought the most important thing was to "watch the hazards carefully" if we did not want to upset the balance between risk and benefit. GMAG he thought was flexible enough to do this. 